\title{
Current Distribution Analysis for High Temperature Superconducting Cable Considering Hysteresis Characteristics
}

\author{
Shuhong WANG ${ }^{\mathrm{a}}$, Jie QIU ${ }^{\mathrm{a}}$, Xinying LIU ${ }^{\mathrm{a}}$, Zhixuan WANG ${ }^{\mathrm{a}}$, Jianguo ZHU ${ }^{\mathrm{b}}$, Youguang \\ GUO $^{\mathrm{b}}$, Zhi Wei LIN ${ }^{\mathrm{b}}$, Yi WANG ${ }^{\mathrm{b}}$ and Wei XU' \\ ${ }^{\text {a }}$ Faculty of Electrical Engineering, Xi'an Jiaotong University, Xi'an 710049, China \\ Tel.: +86 29 82668630-213; E-mail: shwang@mail.xjtu.edu.cn \\ ${ }^{\mathrm{b}}$ School of Electrical, Mechanical and Mechatronic Systems, University of Technology, Sydney, \\ NSW 2007, Australia
}

\begin{abstract}
This paper presents a hysteresis model for Type-II high temperature superconductor (HTS) by using simplified Preisach Model, in which the Preisach distribution function $\mu_{k}(\alpha, \beta)$ is determined only based on the $B$ $H$ limiting loop. The nonlinear dynamic circuit model of superconductor is established. In the circuit model, the hysteresis inductance and hysteresis loss described by simplified Preisach Model are deduced. Applying the hysteresis circuit model, the currents flowing in different superconductor layers of high temperature superconducting cable are simulated, as well as the hysteresis loss of the superconducting cable. The simulation results are verified by comparison with data recorded in literatures. Finally, the influences of hysteresis on superconducting cable are analyzed and discussed.
\end{abstract}

Keywords. Superconducting cable, Hysteresis, Preisach Model, Current distribution

\section{Introduction}

HTS cables for large current transmission in general have a multi-layer structure consisting of parallel connected tapes, twisted in each layer. A warm-dielectric HTS cable is shown in Fig. 1. The control of current distribution among these layers is an important issue for design and optimization of an HTS cable because this significantly affects the current transmission capacity and power losses. The pinned magnetic flux constitutes a memory that gives rise to a hysteresis with corresponding losses and produces an additional voltage in the HTS cable.

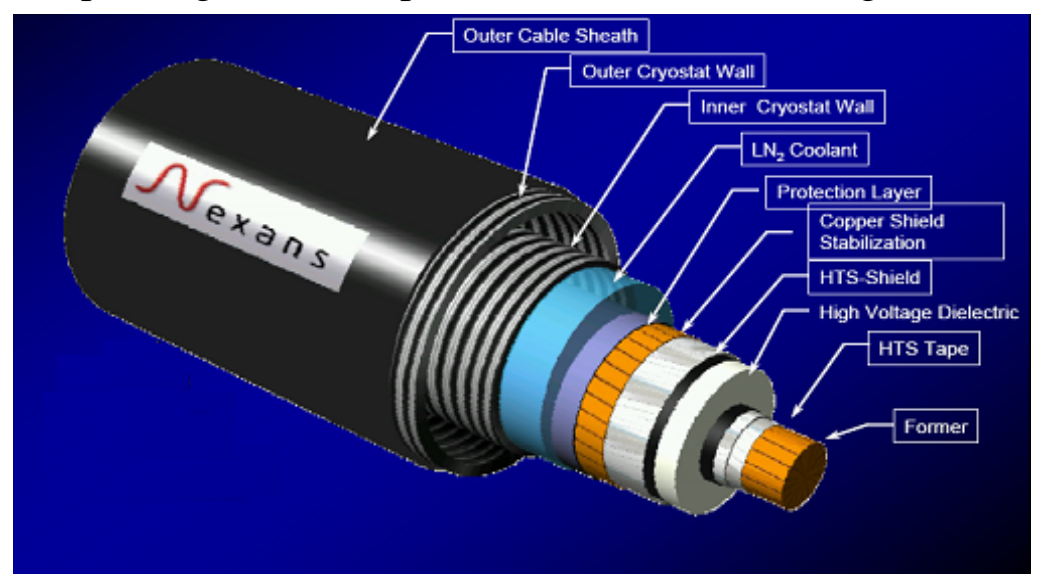

Fig. 1. A HTS cable.

A basic property of the magnetic material is the vector B-H relationship, known as the vector magnetic hysteresis, which describes the phenomenon that the variation of $\mathrm{B}$ lags that 
of $\mathrm{H}$ in either magnitude or direction when the rate of variation is very slow. Owing to the very complex mechanisms of magnetic hysteresis, which is not yet fully understood so far, the development of mathematical models of magnetisation processes has not been successful especially for engineering applications despite the fact that many attempts have been made by various researchers. By now, various models have been developed with a better understanding of magnetic hysteresis phenomenon. Among them, the most noticeable works are the Stoner and Wohlfarth (S-W) model [19], the Preisach model [20] and the combined model [21]. In 1948, Stoner and Wohlfarth postulated a theory based on the rotation of magnetic moments of single domain particles with respect to their easy axes. In 1990, Atherton and Beattie [22] suggested a modification to incorporate particle interactions into the S-W model by the addition of a mean field term, but the numerical implementation and experimental verification of the theory was not reported. The Preisach model has been greatly improved and generalised to model dynamic hysteresis [23], which accounts for the influence of the rate of variation of magnetic field strength on branching, and magnetisation dependent hysteresis [24,25], which assumes that the magnetisation for a given field strength depends on both the previous magnetic history and the current value of magnetisation. The Preisach model has also been generalised to represent vector hysteresis [26]. The vector hysteresis is simulated by a set of scalar Preisach models, each having a different magnetisation direction and responding to the component of the applied field in that direction.

The Preisach model has been used to describe the hysteresis in superconducting cable [1]. J. G. Zhu, et al. proposed the circuit model for magnetic core by using simplified hysteresis Preisach model [2].

In this paper, the simplified Preisach model proposed in [2] is applied to describe the magnetic performance of type-II superconductors in Section II. In Section III, the hysteresis inductance is deduced according to the simplified model. The nonlinear dynamic circuit model considering hysteresis inductance is presented. The circuit equations are solved by using Back-Eular method and Newton-Raphson iteration. In Section IV, comparison between the simulated results in [1] is conducted and discussed.

\section{Simplified Preisach Model}

To model the nonlinear magnetization property and the hysteresis loss, the Preisach model is adopted for its parameter identification and considerable accuracy. However, distribution function $\mu(\alpha, \beta)$ is difficult to be determined. Statistic method is applied to calculate the distribution function according to a mass of repeatable experiments. In [2], a simplified method is proposed to deduce the distribution funtion only based on the measured limiting hysteresis loop. Fig. 2 shows the hysteresis loop of Bi-3222 and the general Preisach diagram.
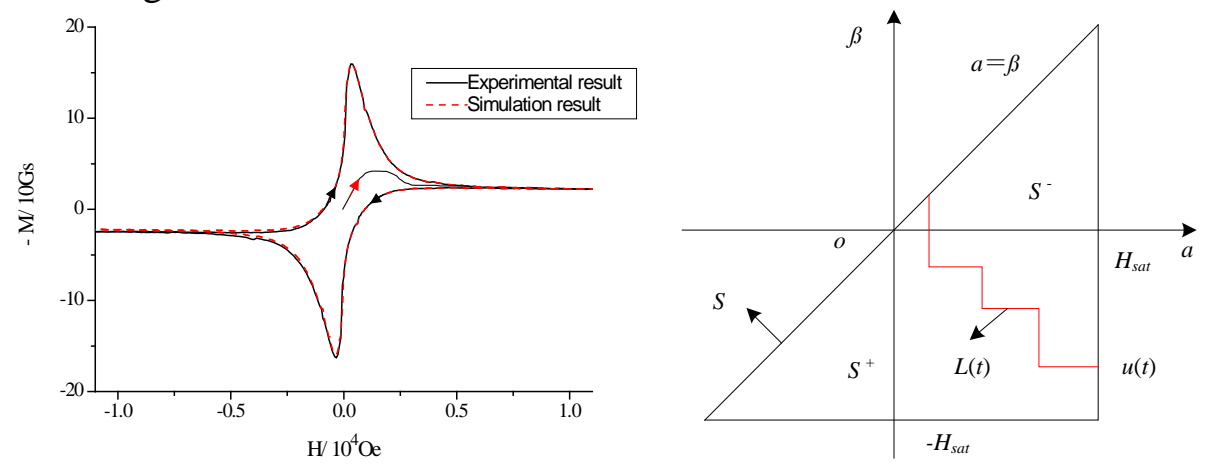

Fig. 2. (a) Hysteresis loop of Bi-3222 and (b) General Preisach diagram. 
In Fig. 2(b), in the triangle $\mathrm{S},-H_{\text {sat }} \leq \alpha \leq \beta \leq H_{\text {sat }}, H_{\text {sat }}$ is the saturation magnetic field strength.

In simplified Preisach model, when a magnetic material is magnetized from the initial unmagnetized state, the flux density $B$ due to magnetic field $H$ is expressed as

$$
\begin{aligned}
B(t) & =\hat{\Gamma} H(t)=\iint_{\alpha \geq \beta} \mu(\alpha, \beta) \hat{\gamma}_{\alpha \beta}(H(t)) \cdot d \alpha d \beta \\
& =\iint_{S^{+}} \mu(\alpha, \beta) d \alpha d \beta-\iint_{S^{-}} \mu(\alpha, \beta) d \alpha d \beta=T(H,-H)
\end{aligned}
$$

where, in the triangle S, $\hat{\gamma}_{\alpha \beta}$ is a sign function, $\hat{\gamma}_{\alpha \beta}(\mathrm{H})$ is equal to 1 on enclosed area S+ and is -1 on $\mathrm{S}^{-}$. The interface between $\mathrm{S}^{+}$and $\mathrm{S}^{-}, L(t)$, is deduced by the history of magnetization.

$T(\alpha, \beta)$, the area integration of hysteresis distribution function, is discribed as

$$
T(\alpha, \beta)=\int_{\beta}^{\alpha} \int_{y}^{\alpha} \mu(\alpha, \beta) d x d y=\int_{\beta}^{\alpha} \int_{\beta}^{x} \mu(\alpha, \beta) d x d y
$$

On a downward trajectory of limiting $B-H$ loop, (1) is rewritten as

$$
B_{d}(H)=\left[\iint_{S^{+}} \mu(\alpha, \beta) d \alpha d \beta-\iint_{S^{-}} \mu(\alpha, \beta) d \alpha d \beta\right]=B\left(H_{\text {sat }}\right)-2 T\left(H_{\text {sat }}, H\right)
$$

On a upward trajectory, (1) is can be derived as

$$
B_{u}(H)=\left[\iint_{S^{+}} \mu(\alpha, \beta) d \alpha d \beta-\iint_{S^{-}} \mu(\alpha, \beta) d \alpha d \beta\right]=-B\left(H_{\text {sat }}\right)+2 T\left(H,-H_{\text {sat }}\right)
$$

$T(\alpha, \beta)$ can be rewritten as

$$
T(\alpha, \beta)=\frac{B_{d}(\beta)-B_{u}(\alpha)}{2}+F(\alpha) F(-\beta)
$$

where, $B_{d}$ and $B_{u}$, are the flux densities on the downward and upward trajectories of the limiting $B$ - $H$ loop, and

$$
\begin{aligned}
& F(H)=\frac{B_{u}(H)-B_{d}(H)}{2 \sqrt{B_{d}(H)}} \quad(\text { when } H \geq 0) \\
& F(H)=\sqrt{B_{d}(-H)} \quad(\text { when } H<0)
\end{aligned}
$$

It should be noted that $F(a)$ is expressed only in terms of flux density values of the $B-H$ limiting loop with out considering the distribution function. Thus, only the limiting $B-H$ loop is needed for the implementation of the hysteresis model.

Considering the diamagnetism of superconductor, Eq. (1) may be rewritten as

$$
M(t)=\hat{\Gamma} H(t)=-\iint_{\alpha \geq \beta} \mu(\alpha, \beta) \hat{\gamma}_{\alpha \beta}(H(t)) \cdot d \alpha d \beta
$$

In Fig. 1(a), the simulated hysteresis loop by using simplified preisach model is similar to experimental loop of Bi-32222, a kind of type-II superconducting material..

The hysteresis loss can be calculated as

$$
Q=\iint_{\Omega} \mu(\alpha, \beta)(\alpha-\beta) d \alpha d \beta
$$




\section{Hysteresis Inductance Model}

The inductance of the superconducting tapes is derived based on simplified Preisach model [1].

In a HTS cable, the axial magnetic flux linkage of the ith Layer, $\varphi_{z, i}$, can be calculated as

$$
\begin{aligned}
\varphi_{z, i} & =\iint_{\Gamma} B_{z, i} \cdot n_{z, i} d S=\int_{r_{1}}^{r_{2}} 2 \pi \rho f\left(\frac{I_{i}}{2 \pi r_{i}} \tan \beta_{i} \frac{\left(r_{i}+d\right)-\rho}{\left(r_{i}+d\right)-r_{i}}\right) d \rho \cdot \frac{1}{L_{i}} \\
& =\int_{r_{1}}^{r_{2}} \frac{\rho}{r_{i}} \tan \beta_{i} \cdot f\left(\frac{I_{i}}{2 \pi r_{i}} \tan \beta_{i} \frac{\left(r_{i}+d\right)-\rho}{\left(r_{i}+d\right)-r_{i}}\right) d \rho
\end{aligned}
$$

and the circumferential axial magnetic flux linkage $\varphi_{\theta, i}$ is

$$
\varphi_{\theta, i}=\iint_{\Gamma} B_{\theta, i} \cdot n_{\theta, i} d S=\int_{r_{1}}^{r_{2}} f\left(\frac{I_{i}}{2 \pi \rho} \cdot \frac{\rho^{2}-r_{i}^{2}}{\left(r_{i}+d\right)^{2}-r_{i}^{2}}\right) d \rho
$$

where, $B_{z, i}$ and $B_{\theta, i}$ are the axial and circumferential axial magnetic flux densities of the $i$ th layer, respectively. $f(x)$ is the simplified Preisach model. $I_{i}$ is the current flowing through the $i$ th superconducting layer. $r_{i}, \beta_{i} L_{i}$ and $d$ are the radius, tape pitch angle, length and thickness of the $i$ th superconducting layer, respectively.

The integration can be calculated by using Gauss-Legendre method.

The total magnetic flux linkage $\varphi_{i}$ is

$$
\varphi_{i}=\varphi_{z, i}+\varphi_{\theta, i}
$$

Thus, the hysteresis inductance of the ith superconducting layer $L_{s, i}$ is

$$
L_{s, i}=\frac{\mathrm{d} \varphi_{i}}{\mathrm{~d} I_{i}}
$$

\section{Nonlinear Dynamic circuit Model Considering Hysteresis}

The modified equivalent circuit of multi-layer high superconducting cable is shown in Fig. 3 [1][3]. The determination of self-, mutual- inductances and nonlinear resistances superconducting layers may refer to [3].

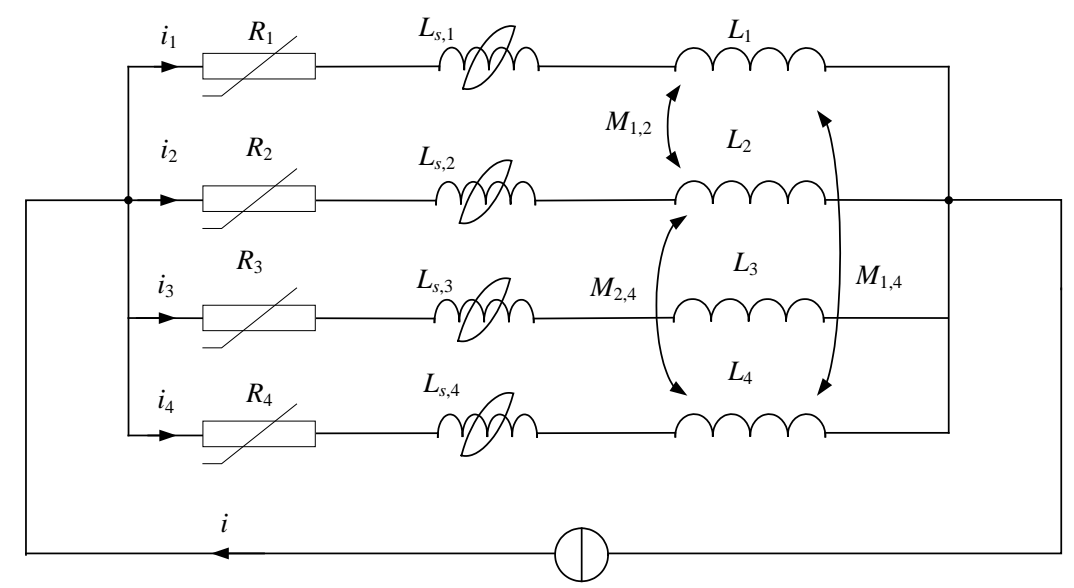


Fig. 3. A equivalent circuit of HTS cable considering hysteresis.

The circuit equations can be deduced as

$$
\left\{\begin{array}{l}
R_{1} i_{1}(t)+L_{1} \frac{d i_{1}(t)}{d t}+L_{s, 1} \frac{d i_{1}(t)}{d t}+M_{12} \frac{d i_{2}(t)}{d t}+\cdots+M_{1 n} \frac{d i_{n}(t)}{d t}=U(t) \\
M_{21} \frac{d i_{1}(t)}{d t}+R_{2} i_{2}(t)+L_{2} \frac{d i_{2}(t)}{d t}+L_{s, 2} \frac{d i_{2}(t)}{d t}+\cdots+M_{2 n} \frac{d i_{n}(t)}{d t}=U(t) \\
\vdots \quad \vdots \quad \ddots \quad \vdots \quad \vdots \\
M_{n 1} \frac{d i_{1}(t)}{d t}+M_{n 2} \frac{d i_{2}(t)}{d t}+\cdots+R_{n} i_{n}(t)+L_{n} \frac{d i_{n}(t)}{d t}+L_{s, n} \frac{d i_{n}(t)}{d t}=U(t) \\
i_{1}(t)+i_{2}(t)+i_{3}(t)+\cdots+i_{n}(t)=i(t)
\end{array}\right.
$$

These nonlinear differential equations may be solved by using Newton-Raphson method.

\section{Example}

In order to verify the correction of simplified Preisach model, the superconducting cable prototypes, which is simulated in [1], is employed. Table I lists the structural parameters of these prototypes. Bi-3222 is adopted as the superconductor.

Table I

Structural parameters of superconducting cable prototypes

\begin{tabular}{|c|c|c|c|c|c|c|}
\hline & $\begin{array}{l}\text { Peak of } \\
\text { Current }\end{array}$ & No. of Layer & 1 & 2 & 3 & 4 \\
\hline \multirow{3}{*}{ Prototype a } & \multirow{3}{*}{$I_{p}=3800 \mathrm{~A}$} & $r_{i} / \mathrm{mm}$ & 19.88 & 20.60 & 21.32 & 22.07 \\
\hline & & $\theta_{i} /^{\mathrm{o}}$ & 15 & 30 & 25 & 10 \\
\hline & & Orientation & +1 & +1 & +1 & +1 \\
\hline \multirow{3}{*}{ Prototype b } & \multirow{3}{*}{$I_{p}=3800 \mathrm{~A}$} & $r_{i} / \mathrm{mm}$ & 19.88 & 20.60 & 21.32 & 22.07 \\
\hline & & $\theta_{i}{ }^{\circ}$ & 35 & 35 & 5 & 5 \\
\hline & & Orientation & +1 & +1 & -1 & -1 \\
\hline \multirow{3}{*}{ Prototype c } & \multirow{3}{*}{$I_{p}=4800 \mathrm{~A}$} & $r_{i} / \mathrm{mm}$ & 19.88 & 20.60 & 21.32 & 22.07 \\
\hline & & $\theta_{i} /^{0}$ & 25 & 10 & 13 & 36 \\
\hline & & Orientation & +1 & +1 & -1 & -1 \\
\hline \multirow{3}{*}{ Prototype d } & \multirow{3}{*}{$I_{p}=4800 \mathrm{~A}$} & $r_{i} / \mathrm{mm}$ & 19.88 & 20.60 & 21.32 & 22.07 \\
\hline & & $\theta_{i} /^{\mathrm{o}}$ & 32 & 14 & 18 & 34 \\
\hline & & Orientation & +1 & +1 & +1 & +1 \\
\hline
\end{tabular}

$\left.{ }^{2000}\right]$ curtent results simutated in [1] and by the presented method in this paper are pictured

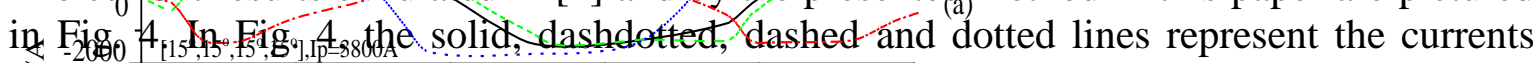
flawing through Layer 1, 2, 3 and 4, respectively. It can be seen that the results calculated b the simplified Preisach model is similar to those presented in [1].

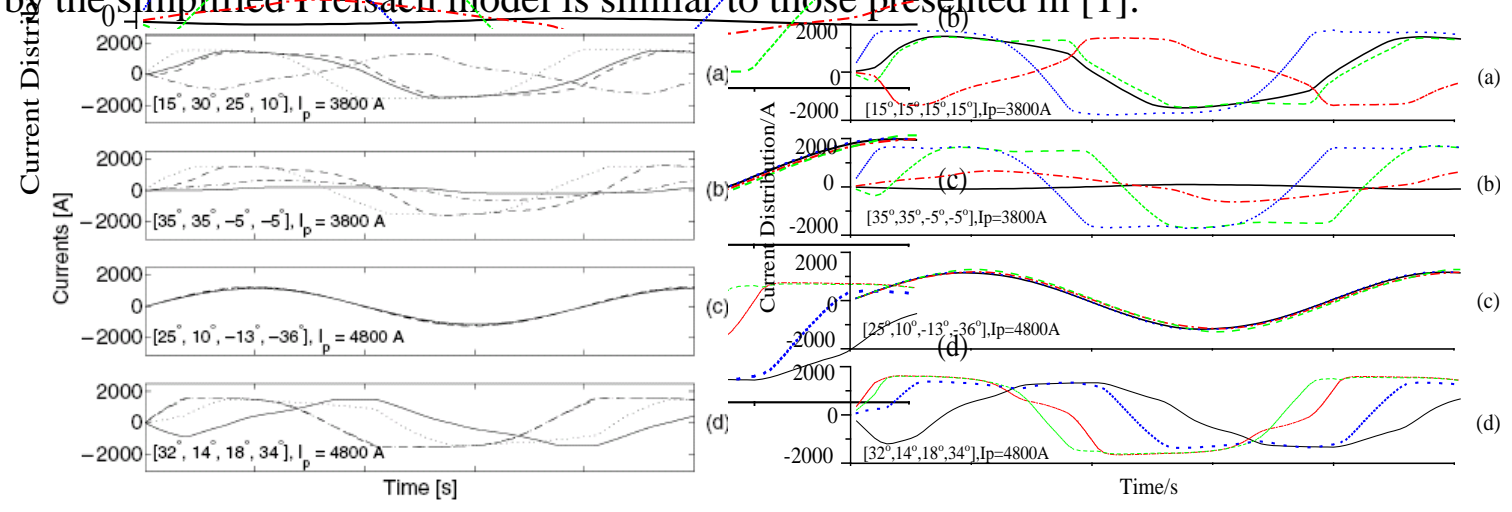



(a) Currents simulated in [1]
(b) Currents simulated in this paper

Fig. 4. The simulated currents.

Fig. 5. illustrates the current distribution of the 4-layer HTS cable, the magnetic hystersis loop of the 1st layer and the hysteresis loss with total current 4800A, tape pitch angles of layers $+25^{\circ},+10^{\circ},-14^{\circ}$ and $-37^{\circ}$, respectively. In this case, the current distribution becomes uniform. At static operation, the hysteresis loss per cycle approaches to a constant.

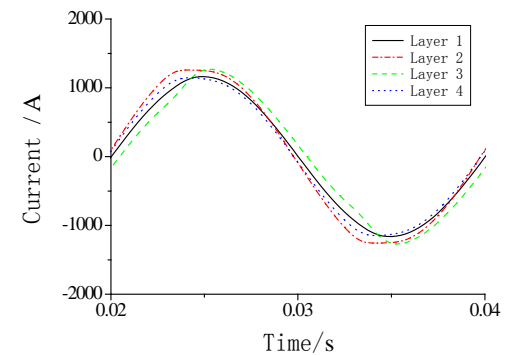

(a) Current distribution

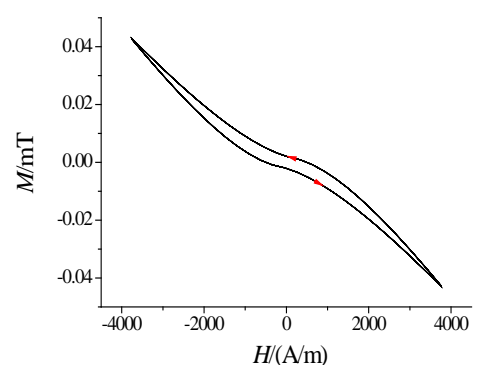

(b) Hysteresis loop

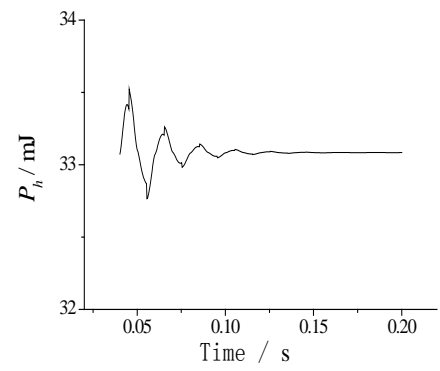

(c) Hysteresis loss

Fig. 5. The simulated results of a 4-layer HTS cable.

\section{Conclusion}

The hysteresis of superconductor causes the non-uniform current distribution in HTS cable. In this paper, a simplified Preisach model, which just considers the limiting loop of hysteresis loop, is applied to describe the magnetic hysteresis performance of superconducting cable. The hysteresis loss is presented based on the simplified model. The circuit model of HTS cable considering hysteresis is deduced. Comparison with the results in literatures, the correction of this simplified Preisach model and circuit model is verified. Choosing proper structure parameters, the current distribution of HTS layer can be uniform.

\section{References}

[19] E.C. Stoner and E.P. Wohlfarth, “A mechanism of magnetic hysteresis in heterogeneous alloys”, Phil. Trans. Roy. Soc., vol. 240A, May 1948, pp. 599-642.

[20] V.F. Preisach, “Uber die magnetische Nachwirkung”, Z. fur Physik, Bd. 94, 1935, pp. 277-302.

[21] J. Oti and E.D. Torre, “A vector moving model of both reversible and irreversible magnetising processes”, J. Appl. Phys., vol. 67, no. 9, May 1990, pp. 5364-5366.

[22] D.L. Atherton, and J.R. Beattie, "A mean field Stoner-Wohlfarth hysteresis model", IEEE Trans. Magn., vol. 26, no. 6, Nov. 1990, pp. 3059-3063 .

[23] I.D. Mayergoyz, "Dynamic Preisach models of hysteresis", IEEE Trans. Magn., vol. 24, no. 6, Nov. 1988, pp. 2925-2927.

[24] J. Oti, F. Vajda, and E.D. Torre, “Identification of parameters in a moving model”, J. Appl. Phys., vol. 69, no. 8, Apr. 1991, pp. 4826-4828.

[25] E.D. Torre, "Existence of magnetisation-dependent Preisach models", IEEE Trans. Magn., vol. 27, no. 4, July 1991, pp. 3697-3699.

[26] A.A. Adly and I.D. Mayergoyz, “A new vector Preisach-type model of hysteresis”, J. Appl. Phys., vol. 73, no. 10, May 1993, pp. 5824-5826.

[1] F. Grilli and M. Sjostrom, "Prediction of resistive and hysteretic losses in a multi-layer high- Tc superconducting cable,” Superconductor Science and Technology, 17 (2004), 409-416.

[2] S. Y. R. Hui, Jian Guo Zhu, and V. S. Ramsden. "A generalized dynamic circuit model of magnetic cores for low- and high-frequency applications. II. Circuit model formulation and implementation”. IEEE Transactions on POWER ELECTRONICS, 11 (1996), 251-259

[3] Shuhong Wang, Jie Qiu, Zhen Zhao, Jian Guo Zhu and Youguang Guo. "Structural Parameter Design on Multilayer Conductors of HTS AC Transmission Cable by Means of Particle Swarm Optimization”. Journal of the Japan Society of Applied Electromagnetics and Mechanics, Vol.I5, No.3 (2007), 290-293 